\title{
Perturbation correction for the free energy and structure of simple fluid mixtures
}

\author{
F. Lado \\ Department of Physics, North Carolina State University, Raleigh, North Carolina 27607
}

(Received 24 July 1973)

\begin{abstract}
Adapting an analysis due to Morita and Hiroike and Green which led to the hypernetted-chain (HNC) integral equation, we obtain an expression for the difference in free energy between a perturbed mixture with potentials $\phi_{\alpha \beta}=\phi_{\alpha \beta}^{(0)}+\phi_{a \beta}^{(1)}$ and a reference mixture with potentials $\phi_{\alpha \beta}^{(0)}$, a result which is rendered computable by an HNC-type approximation. No further approximations are required to compute the corrected pair distribution functions of the perturbed mixture, but a proposed second approximation yields a particularly simple solution. This work generalizes earlier results on single-component fluids.
\end{abstract}

\section{INTRODUCTION}

This work generalizes, for the case of classical fluid mixtures, previous results obtained for single-component fluids. ${ }^{1,2}$ As in these earlier papers, we shall assume that the properties of a reference, or unperturbed, system, defined by the short-range, repulsive potentials $\varphi_{\alpha \beta}^{(0)}(r)$ between molecular species $\alpha$ and $\beta$, are known. Given this knowledge about the reference system, the general question posed by perturbation theory is how such information can be used to calculate the properties of the perturbed system with potentials

$$
\varphi_{\alpha \beta}(r)=\varphi_{\alpha \beta}^{(0)}(r)+\varphi_{\alpha \beta}^{(1)}(r) .
$$

The answer given here is in two parts. In Sec. II, we obtain an expression for the difference in free energy between the perturbed and reference systems. The derivation, which generalizes analyses by Morita and Hiroike ${ }^{3}$ and Green ${ }^{4}$ which led to the hypernetted-chain ${ }^{3-5}$ (HNC) integral equation, produces an expression which is similarly rendered computable after an approximation of the HNC type. The free energy correction depends on, and by a minimal principle implies equations for, the corrected pair distribution functions $g_{\alpha \beta}(r)$. These equations are discussed in Sec. III, where it is pointed out that they can be solved iteratively with no further approximations, but that a particularly simple second approximation can, if desired, yield a solution immediately.

These results differ from other perturbational or variational expressions for the corrected thermodynamic properties of mixtures ${ }^{6}$ in that they do not involve a truncated series expansion. Instead, an infinite number of selected terms is in effect summed. On the other hand, the computational effort required to produce final numbers, while conceptually uncomplicated, is greater than for the conformal theories such as the van der Waals model ${ }^{7,8}$ and the random approximation, ${ }^{8}$ though of course much depends on the choice of reference system and possible further simplifying approximations.

The Percus-Yevick ${ }^{9}$ (PY) and HNC integral equations, generalized for mixtures, offer another alternative to the determination of mixture properties, ${ }^{10}$ again at the cost of increased computation. Both these equations are of course approximate, but it is known that of the two the PY equation is superior, and reasonably accurate, for potentials possessing a sharp, repulsive core, ${ }^{11}$ while the opposite is apparently the case for potentials lacking such a core. ${ }^{12}$ This suggests that, with the separation effected in Eq. (1), the PY equation could be advantageously used to study the reference system, and the corrections incorporated through the HNC-type equations discussed here; in effect, tailoring the problem to suit the available approximate techniques.

\section{CORRECTION FOR THE HELMHOLTZ FREE ENERGY}

To allow greater explicitness in the results, we shall consider here a binary mixture containing $N_{1}$ molecules of type 1 and $N_{2}$ molecules of type 2. The formal extension to arbitrary number of components is straightforward.

Using canonical ensemble formalism, we take the $N=N_{1}+N_{2}$ molecules to be in thermal equilibrium, in a volume $V$, at the temperature $T$ $=\left(k_{B} \beta\right)^{-1}$. For a given configuration, the potential energy of the mixture is written

$$
U\left(\mathbf{r}^{N} ; \xi\right)=U^{(0)}\left(\mathbf{r}^{N}\right)+\xi U^{(1)}\left(\mathbf{r}^{N}\right),
$$

where

$$
\begin{aligned}
U^{(m)}\left(\mathrm{r}^{N}\right)= & \sum_{1 \leqslant i<j \leqslant N_{1}} \varphi_{11}^{(m)}\left(r_{i j}\right)+\sum_{\substack{1 \leqslant i \leqslant N_{1} \\
N_{1}+1 \leqslant j \leqslant N}} \varphi_{12}^{(m)}\left(r_{i j}\right) \\
& +\sum_{N_{1}+1 \leqslant i<j \leqslant N} \varphi_{22}^{(m)}\left(r_{i j}\right),
\end{aligned}
$$


for $m=0,1$. In Eq. (2), we have used the potential separation of (1) and in addition have introduced a "charging" parameter $\xi$ whose value will eventually be unity. The reference system corresponds to $\xi=0$ and quantities pertaining to it will be labeled with a superscript 0 .

We are interested in the configurational Helmholtz free energy

$$
\begin{aligned}
& A^{C}(\xi)=-k_{B} T \ln Q(\xi), \\
& Q(\xi) \equiv V^{-N} \int d^{3 N} r \exp \left[-\beta U\left(\mathbf{r}^{N} ; \xi\right)\right],
\end{aligned}
$$

and shall employ in its determination a generalization of the work of Morita and Hiroike ${ }^{3}$ and Green. ${ }^{4}$ Introduction of the charging parameter $\xi$ allows us now to formally write for the difference in free energy between arbitrarily "charged" and reference systems

$$
\begin{aligned}
A(\xi)-A^{(0)}= & \int_{0}^{\xi} d \xi d A^{c}(\xi) / d \xi \\
= & \frac{1}{2} N \rho k_{B} T \int_{0}^{\xi} d \xi \int d \mathbf{r} \sum_{\alpha, \beta} x_{\alpha} x_{\beta} g_{\alpha \beta} \\
& \times(r ; \xi) \beta \varphi_{\alpha \beta}^{(1)}(r),
\end{aligned}
$$

where $\rho$ is the overall number density $N / V$ and

$$
\begin{aligned}
x_{\alpha}=N_{\alpha} / N= & \rho_{\alpha} / \rho, \\
g_{\alpha \beta}(r ; \xi)= & {\left[N_{\alpha}\left(N_{\beta}-\delta_{\alpha \beta}\right) / \rho_{\alpha} \rho_{\beta} Q(\xi)\right] \int d^{3(N-2)} r } \\
& \times \exp \left[-\beta U\left(\mathbf{r}^{N} ; \xi\right)\right] .
\end{aligned}
$$

The integral in (8) is over the positions of all but two particles, of types $\alpha$ and $\beta$, separated by the distance $r$; the indices $\alpha$ and $\beta$, as mentioned above, take on just the values 1 and 2 in the present application.

To analyze the integrand of (6) we shall use results from the graphical analysis of the pair distribution function of single-component systems. ${ }^{3-5,13}$ The extension to mixtures is evident. Thus, $S_{\alpha \beta}(r ; \xi)$ shall be the sum of all series-type graphs which begin on a molecule of type $\alpha$ and end on a molecule of type $\beta$. That is, the two root points of each diagram are occupied by molecules of type $\alpha$ and $\beta$. Similarly, $B_{\alpha \beta}(r ; \xi)$ is the sum of bridgetype diagrams with these same root points. It should be noted that intervening field points in these diagrams can belong to molecules of any type. In terms of the series and bridge sets, it is known that one may write $g_{\alpha \beta}$ as

$$
g_{\alpha \beta}(r ; \xi)=\exp \left[-\beta \varphi_{\alpha \beta}(r ; \xi)+S_{\alpha \beta}(r ; \xi)+B_{\alpha \beta}(r ; \xi)\right],
$$

where $\varphi_{\alpha \beta}(r ; \xi)$ is just Eq. (1) with the insertion of the charging parameter,

$$
\varphi_{\alpha \beta}(r ; \xi)=\varphi_{\alpha \beta}^{(0)}(r)+\xi \varphi_{\alpha \beta}^{(1)}(r) .
$$

With $\xi$ set to zero, we get the reference system version of Eq. (9),

$$
g_{\alpha \beta}^{(0)}(r)=\exp \left[-\beta \varphi_{\alpha \beta}^{(0)}(r)+S_{\alpha \beta}^{(0)}(r)+B_{\alpha \beta}^{(0)}(r)\right],
$$

which, divided into (9), gives a formal expression for the perturbed $g_{\alpha \beta}$,

$$
\begin{aligned}
g_{\alpha \beta}(r ; \xi)= & g_{\alpha \beta}^{(0)}(r) \exp \left[-\xi \beta \varphi_{\alpha \beta}^{(1)}(r)+\Delta S_{\alpha \beta}(r ; \xi)\right. \\
& \left.+\Delta B_{\alpha \beta}(r ; \xi)\right] .
\end{aligned}
$$

In this equation,

$$
\Delta S_{\alpha \beta}(r ; \xi) \equiv S_{\alpha \beta}(r ; \xi)-S_{\alpha \beta}^{(0)}(r)
$$

and

$$
\Delta B_{\alpha \beta}(r ; \xi) \equiv B_{\alpha \beta}(r ; \xi)-B_{\alpha \beta}^{(0)}(r)
$$

are the differences in the series and bridge sets resulting from turning on the perturbing potentials $\varphi_{\alpha \beta}^{(1)}$.

The integrand of (6) can now be re-expressed by differentiating (12). This gives

$$
\begin{aligned}
g_{\alpha \beta}(r ; \xi) \beta \varphi_{\alpha \beta}^{(1)}(r)= & -\partial g_{\alpha \beta}(r ; \xi) / \partial \xi \\
& +g_{\alpha \beta}(r ; \xi) \partial\left[\Delta S_{\alpha \beta}(r ; \xi)\right. \\
& \left.+\Delta B_{\alpha \beta}(r ; \xi)\right] / \partial \xi \\
= & -(\partial / \partial \xi)\left\{g_{\alpha \beta}(r ; \xi)\right. \\
& -g_{\alpha \beta}(r ; \xi) \xi \beta \varphi_{\alpha \beta}^{(1)}(r) \\
& \left.-g_{\alpha \beta}(r ; \xi) \ln \left[g_{\alpha \beta}(r ; \xi) / g_{\alpha \beta}^{(0)}(r)\right]\right\} \\
& -\Delta S_{\alpha \beta}(r ; \xi) \partial g_{\alpha \beta}(r ; \xi) / \partial \xi \\
& -\Delta B_{\alpha \beta}(r ; \xi) \partial g_{\alpha \beta}(r ; \xi) / \partial \xi .
\end{aligned}
$$

The series set $S_{\alpha \beta}$ is now eliminated in favor of the direct correlation function $C_{\alpha \beta}$, defined by

$$
C_{\alpha \beta}(r ; \xi)=G_{\alpha \beta}(r ; \xi)-S_{\alpha \beta}(r ; \xi),
$$

or, after subtracting the equivalent reference system equation,

$$
\Delta C_{\alpha \beta}(r ; \xi)=\Delta G_{\alpha \beta}(r ; \xi)-\Delta S_{\alpha \beta}(r ; \xi),
$$

where

$$
G_{\alpha \beta}(r ; \xi) \equiv g_{\alpha \beta}(r ; \xi)-1
$$

and $\Delta G_{\alpha \beta}, \Delta C_{\alpha \beta}$ are defined as in Eq. (13). With the use of (16), Eq. (14) is rewritten to read

$$
\begin{aligned}
& g_{\alpha \beta}(r ; \xi) \beta \varphi_{\alpha \beta}^{(1)}(r) \\
&=--(\partial / \partial \xi)\left\{\frac{1}{2} \Delta G_{\alpha \beta}(r ; \xi)^{2}+g_{\alpha \beta}(r ; \xi)\right. \\
&-g_{\alpha \beta}(r ; \xi) \xi \beta \varphi_{\alpha \beta}^{(1)}(r)-g_{\alpha \beta}(r ; \xi) \\
&\left.-\ln \left[g_{\alpha \beta}(r ; \xi) / g_{\alpha \beta}^{(0)}(r)\right]\right\}+\Delta C_{\alpha \beta}(r ; \xi) \partial \Delta G_{\alpha \beta} \\
& \times(r ; \xi) / \partial \xi-\Delta B_{\alpha \beta}(r ; \xi) \partial \Delta G_{\alpha \beta}(r ; \xi) / \partial \xi,
\end{aligned}
$$

which, inserted into (6), gives

$$
\begin{aligned}
& \beta\left[A(\xi)-A^{(0)}\right] / N \\
& \quad=\frac{1}{2} \rho \int d r \sum_{\alpha, \beta} x_{\alpha} x_{B}\left\{g_{\alpha \beta}(r ; \xi) \xi \beta \varphi_{\alpha \beta}^{(1)}(r)\right.
\end{aligned}
$$




$$
\begin{aligned}
& +g_{\alpha \beta}(r ; \xi) \ln \left[g_{\alpha \beta}(r ; \xi) / g_{\alpha \beta}^{(0)}(r)\right]-\Delta G_{\alpha \beta}(r ; \xi) \\
& \left.-\frac{1}{2} \Delta G_{\alpha \beta}(r ; \xi)^{2}\right\}+\frac{1}{2} \rho \int_{0}^{\xi} d \xi \int d \mathrm{r} \sum_{\alpha, \beta} x_{\alpha} x_{\beta} \Delta C_{\alpha \beta} \\
& \times(r ; \xi) \partial \Delta G_{\alpha \beta}(r ; \xi) / \partial \xi-\frac{1}{2} \rho \int_{0}^{\xi} d \xi \int d \mathbf{r} \\
& \times \sum_{\alpha, \beta} x_{\alpha} x_{\beta} \Delta B_{\alpha \beta}(r ; \xi) \partial \Delta G_{\alpha \beta}(r ; \xi) / \partial \xi
\end{aligned}
$$

Further evaluation of the penultimate term in (19) can be carried out by introducing the generalized Ornstein-Zernike equation which reads, in Fourier transform notation,

$\tilde{G}_{\alpha \beta}(k ; \xi)=\tilde{C}_{\alpha \beta}(k ; \xi)+\sum_{\gamma} \rho_{\gamma} \tilde{C}_{\alpha \gamma}(k ; \xi) \tilde{G}_{\gamma \beta}(k ; \xi)$.

This set of simultaneous equations can be solved for $\tilde{C}_{\alpha \beta}$ or $\tilde{G}_{\alpha \beta}$. In the former case, after some algebra, one gets for a binary mixture (we suppress the function arguments for brevity)

$$
\begin{aligned}
& \tilde{C}_{11}=D_{G}^{-1}\left[\left(1+\rho_{2} \tilde{G}_{22}\right) \tilde{G}_{11}-\rho_{2} \tilde{G}_{12}^{2}\right], \\
& \tilde{C}_{12}=\tilde{C}_{21}=D_{G}^{-1} \tilde{G}_{12}, \\
& \tilde{C}_{22}=D_{G}^{-1}\left[\left(1+\rho_{1} \tilde{G}_{11}\right) \tilde{G}_{22}-\rho_{1} \tilde{G}_{12}^{2}\right],
\end{aligned}
$$

where

$$
D_{G} \equiv\left(1+\rho_{1} \tilde{G}_{11}\right)\left(1+\rho_{2} \tilde{G}_{22}\right)-\rho_{1} \rho_{2} \tilde{G}_{12}^{2} .
$$

The inverse solution is

$$
\begin{aligned}
& \tilde{G}_{11}=D_{C}^{-1}\left[\left(1-\rho_{2} \tilde{C}_{22}\right) \tilde{C}_{11}+\rho_{2} \tilde{C}_{12}^{2}\right], \\
& \tilde{G}_{12}=\tilde{G}_{21}=D_{C}^{-1} \tilde{C}_{12}, \\
& \tilde{G}_{22}=D_{C}^{-1}\left[\left(1-\rho_{1} \tilde{C}_{11}\right) \tilde{C}_{22}+\rho_{1} \tilde{C}_{12}^{2}\right],
\end{aligned}
$$

where

$$
D_{C} \equiv\left(1-\rho_{1} \tilde{C}_{11}\right)\left(1-\rho_{2} \tilde{C}_{22}\right)-\rho_{1} \rho_{2} \tilde{C}_{12}^{2} .
$$

It is easy to show that

$$
D_{G}=D_{C}^{-1} \text {, }
$$

and furthermore that

$$
\begin{aligned}
& \sum_{\alpha, \beta} x_{\alpha} x_{\beta} \Delta \tilde{C}_{\alpha \beta}(k ; \xi) \partial \Delta \tilde{G}_{\alpha \beta}(k ; \xi) / \partial \xi \\
&=\rho^{-2}(\partial / \partial \xi)\left\{\sum_{\alpha, \beta}\left[\delta_{\alpha \beta}-\rho_{\alpha} \tilde{C}_{\alpha \beta}^{(0)}(k)\right]\right. \\
&\left.\times \rho_{\beta} \Delta \tilde{G}_{\alpha \beta}(k ; \xi)-\ln D_{G}(k ; \xi)\right\} .
\end{aligned}
$$

We can now write

$$
\begin{aligned}
& \int_{0}^{\xi} d \xi \int d \mathbf{r} \sum_{\alpha, \beta} x_{\alpha} x_{\beta} \Delta C_{\alpha \beta}(r ; \xi) \partial \Delta G_{\alpha \beta}(r ; \xi) / \partial \xi \\
& =(2 \pi)^{-3} \int_{0}^{\xi} d \xi \int d \mathbf{k} \sum_{\alpha, \beta} x_{\alpha} x_{\beta} \Delta \tilde{C}_{\alpha \beta}(k ; \xi) \partial \Delta G_{\alpha \beta}(k ; \xi) / \partial \xi \\
& =\left[\rho^{2}(2 \pi)^{3}\right]^{-1} \int d \mathbf{k}\left\{\sum_{\alpha, \beta}\left[\delta_{\alpha \beta}-\rho_{\alpha} \tilde{C}_{\alpha \beta}^{(0)}(k)\right]\right. \\
& \left.\times \rho_{\beta} \Delta \bar{G}_{\alpha \beta}(k ; \xi)-\ln \left[D_{G}(k ; \xi) / D_{G}^{(0)}(k)\right]\right\}
\end{aligned}
$$

and so we have finally for the free energy difference between perturbed $(\xi=1)$ and reference $(\xi=0)$ mixtures

$$
\begin{aligned}
\beta \Delta A / N \equiv & \beta\left(A-A^{(0)}\right) / N \\
= & \frac{1}{2} \rho \int d \mathbf{r} \sum_{\alpha, \beta} x_{\alpha} x_{\beta}\left\{g_{\alpha \beta}(r) \beta \varphi_{\alpha \beta}^{(1)}(r)\right. \\
& +g_{\alpha \beta}(r) \ln \left[g_{\alpha \beta}(r) / g_{\alpha \beta}^{(0)}(r)\right]-\Delta G_{\alpha \beta}(r) \\
& \left.-\frac{1}{2} \Delta G_{\alpha \beta}(r)^{2}\right\}+\left[2 \rho(2 \pi)^{3}\right]^{-1} \int d \mathbf{k}\left\{\sum _ { \alpha , \beta } \left[\delta_{\alpha \beta}\right.\right. \\
& \left.\left.-\rho_{\alpha} \tilde{C}_{\alpha \beta}^{(0)}(k)\right] \rho_{\beta} \Delta \tilde{G}_{\alpha \beta}(k)-\ln \left[D_{G}(k) / D_{G}^{(0)}(k)\right]\right\} \\
& -\frac{1}{2} \rho \int_{0}^{1} d \xi \int d \mathbf{r} \sum_{\alpha, \beta} x_{\alpha} x_{\beta} \Delta B_{\alpha \beta}(r ; \xi) \\
& \times \partial \Delta G_{\alpha \beta}(r ; \xi) / \partial \xi .
\end{aligned}
$$

No further progress can be made with the last term in (28), since no simple functional is known relating the bridge set to the pair correlation function.

Equation (28) is the generalization of the free energy expression of Morita and Hiroike ${ }^{3}$ and Green, ${ }^{4}$ for the case of a binary mixture and arbitrary reference system. If we chose the ideal gas as the reference system, (28) would read $\beta A^{C} / N$

$$
\begin{aligned}
= & \frac{1}{2} \rho \int d \mathbf{r} \sum_{\alpha, \beta} x_{\alpha} x_{\beta}\left[g_{\alpha \beta}(r) \beta \varphi_{\alpha \beta}(r)+g_{\alpha \beta}(r) \ln g_{\alpha \beta}(r)\right. \\
& \left.-G_{\alpha \beta}(r)-\frac{1}{2} G_{\alpha \beta}(r)^{2}\right]+\left[2 \rho(2 \pi)^{3}\right]^{-1} \int d \mathbf{k} \\
& \times\left[\sum_{\alpha} \rho_{\alpha} \tilde{G}_{\alpha \alpha}(k)-\ln D_{G}(k)\right]-\frac{1}{2} \rho \int_{0}^{1} d \xi \int d \mathbf{r} \\
& \times \sum_{\alpha, \beta} x_{\alpha} x_{\beta} B_{\alpha \beta}(r ; \xi) \partial G_{\alpha \beta}(r ; \xi) / \partial \xi
\end{aligned}
$$

containing now only the mixtures generalization, so that if, say, $x_{2}$ is set to zero in (29), we recover finally the original expression. ${ }^{3,4}$

The HNC approximation applied to Eq. (29) would consist in neglecting the unknown bridge sets $B_{\alpha \beta}$. For single-component fluids, this has been found to generate accurate results when the potential function involved lacks a sharp, repulsive core. ${ }^{12}$ Since the perturbing potentials $\varphi_{\alpha \beta}^{(1)}$ should be, by construction, of this type, we adopt the $\mathrm{HNC}$ approximation for the differences $\Delta B_{\alpha \beta}$ in Eq. (28). Here, it is not the bridge sets which are being neglected, but rather the changes in them caused by the perturbing potentials $\varphi_{\alpha \beta}^{(1)}$. Evidently, this is a less drastic approximation.

We have then the computable approximate result that the free energy correction is

$$
\begin{aligned}
\beta \Delta A / N \approx & \frac{1}{2} \rho \int d \mathbf{r} \sum_{\alpha, \beta} x_{\alpha} x_{\beta}\left\{g_{\alpha \beta}(r) \beta \varphi_{\alpha \beta}^{(1)}(r)-g_{\alpha \beta}(r) \ln \right. \\
& \left.\times\left[g_{\alpha \beta}(r) / g_{\alpha \beta}^{(0)}(r)\right]-\Delta G_{\alpha \beta}(r)-\frac{1}{2} \Delta G_{\alpha \beta}(r)^{2}\right\} \\
& +\left[2 \rho(2 \pi)^{3}\right]^{-1} \int d \mathbf{k}\left\{\sum_{\alpha, \beta}\left[\delta_{\alpha \beta}-\rho_{\alpha} \tilde{C}_{\alpha \beta}^{(0)}(k)\right] \rho_{\beta}\right. \\
& \left.\times \Delta \tilde{G}_{\alpha \beta}(k)-\ln \left[D_{G}(k) / D_{G}^{(0)}(k)\right]\right\}
\end{aligned}
$$


The free energy being the central quantity for the canonical ensemble, Eq. (28) implies all other properties of the corrected system. Thus, the equilibrium condition is that $A$ be minimum; by varying the $g_{\alpha \beta}(r)$ while holding $g_{\alpha \beta}^{(0)}(r)$ fixed, we find

$$
\begin{aligned}
\delta(\beta \Delta A & / N) \\
= & -\frac{1}{2} \rho \int d \mathbf{r} \sum_{\alpha, \beta} x_{\alpha} x_{\beta}\left\{\Delta G_{\alpha \beta}(r)-\beta \varphi_{\alpha \beta}^{(1)}(r)\right. \\
& \left.-\ln \left[g_{\alpha \beta}(r) / g_{\alpha \beta}^{(0)}(r)\right]\right\} \delta G_{\alpha \beta}(r) \\
& +\left[2 \rho(2 \pi)^{3}\right]^{-1} \int d \mathbf{k}\left\{\sum_{\alpha, \beta}\left[\delta_{\alpha \beta}-\rho_{\alpha} \tilde{C}_{\alpha \beta}^{(0)}(k)\right] \rho_{\beta} \delta \tilde{G}_{\alpha \beta}(k)\right. \\
& -\left[1+\rho_{2} \tilde{G}_{22}(k)\right] \rho_{1} \delta \tilde{G}_{11}(k)+2 \rho_{1} \rho_{2} \tilde{G}_{12}(k) \delta \tilde{G}_{12}(k) \\
& \left.-\left[1+\rho_{1} \tilde{G}_{11}(k)\right] \rho_{2} \delta \bar{G}_{22}(k)\right\}-\frac{1}{2} \rho \int d \mathbf{r} \sum_{\alpha, \beta} \\
& \times x_{\alpha} x_{\beta} \Delta B_{\alpha \beta}(r) \delta G_{\alpha \beta}(r)=-\frac{1}{2} \rho \int d \mathbf{r} \sum_{\alpha, \beta} x_{\alpha} x_{\beta} \\
& \times\left\{\Delta G_{\alpha \beta}(r)-\Delta C_{\alpha \beta}(r)+\Delta B_{\alpha \beta}(r)-\beta \varphi_{\alpha \beta}(r)\right. \\
& \left.-\ln \left[g_{\alpha \beta}(r) / g_{\alpha \beta}^{(0)}(r)\right]\right\} \delta G_{\alpha \beta}(r) .
\end{aligned}
$$

This variation vanishes if

$g_{\alpha \beta}(r)=g_{\alpha \beta}^{(0)}(r) \exp \left[-\beta \varphi_{\alpha \beta}^{(1)}(r)+\Delta S_{\alpha \beta}(r)+\Delta B_{\alpha \beta}(r)\right]$,

which is just our starting point, Eq. (12), with $\xi=1$. [The variation of the last term in (28) is considered explicitly in the Appendix.]

Since (28) is extremal with respect to variations in $g(r)$, its dependence on temperature is through $g_{\alpha \beta}^{\left({ }^{(0)}(\gamma)\right.}$ and the explicitly appearing $\beta$. We find thus for the energy difference

$$
\begin{aligned}
\left(E-E_{0}\right) / N & =\partial(\beta \Delta A / N) / \partial \beta \\
= & \frac{1}{2} \rho \int d \mathbf{r} \sum_{\alpha, \beta} x_{\alpha} x_{\beta} g_{\alpha \beta}(r) \varphi_{\alpha \beta}^{(1)}(r) \\
& +\frac{1}{2} \rho \int d \mathbf{r} \sum_{\alpha, \beta} x_{\alpha} x_{\beta} \Delta G_{\alpha \beta}(r)\left[-\partial \ln g_{\alpha \beta}^{(0)}(r) / \partial \beta\right. \\
& +\partial G_{\alpha \beta}^{(0)}(r) / \partial \beta-\partial C_{\alpha \beta}^{(0)}(r) / \partial \beta \\
& \left.+\partial B_{\alpha \beta}^{(0)}(r) / \partial \beta\right]
\end{aligned}
$$

where we have again used the results of the Appendix. But, from Eq. (11), the bracketed term in the second integrand of (33) is just $\varphi_{\alpha \beta}^{(0)}(r)$. The final result is therefore

$$
\begin{aligned}
\left(E-E_{0}\right) / N= & \frac{1}{2} \rho \int d \mathbf{r} \sum_{\alpha \beta \beta} x_{\alpha} x_{\beta}\left[g_{\alpha \beta}(r) \varphi_{\alpha \beta}^{(1)}(r)\right. \\
& \left.+\Delta G_{\alpha \beta}(r) \varphi_{\alpha \beta}^{(0)}(r)\right]=\frac{1}{2} \rho \int d \mathbf{r} \sum_{\alpha, \beta} x_{\alpha} x_{\beta} \\
& \times\left[g_{\alpha \beta}(r) \varphi_{\alpha \beta}(r)-g_{\alpha \beta}^{(0)}(r) \varphi_{\alpha \beta}^{(0)}(r)\right],
\end{aligned}
$$

as expected. Similarly, the difference in pressures is given by

$$
\begin{aligned}
p-p_{0} & =-\partial \Delta A / \partial V \\
& =-\frac{1}{6} \rho^{2} \int d \mathbf{r} \sum_{\alpha, \beta}\left[g_{\alpha \beta} d \varphi_{\alpha \beta}^{(1)}(r) / d r\right.
\end{aligned}
$$

$$
\left.+\Delta G_{\alpha \beta}(r) d \varphi_{\alpha \beta}^{(0)}(r) / d r\right] .
$$

In obtaining (35), it is convenient to first rewrite (28) using the dimensionless variables $r V^{-1 / 3}$ and $k V^{1 / 3}$.

These familiar results following from Eq. (28) merely confirm the internal consistency of the formalism. However, when the approximation (30) is employed, this consistency is lost. We note first that by the minimal rule, (30) implies the approximate relation

$$
g_{\alpha \beta}(r) \approx g_{\alpha \beta}^{(0)} \exp \left[-\beta \varphi_{\alpha \beta}^{(1)}(r)+\Delta S_{\alpha \beta}(r)\right],
$$

where, as expected, $\Delta B_{\alpha \beta}(r)$ has been neglected. But now, continuing as above, it can be shown (see Appendix) that the derivative with respect to $\beta$ of (30) will not agree with (34) supplemented with (36). Similarly the first equality in (35), using (30), will disagree with the second equality, using (36).

In practice, this breakdown in consistency, common to approximate theories, will not often appear explicitly, since the thermodynamic derivatives will be less convenient to use in numerical calculations and, one may expect, less accurate as well, compared with the integrals containing the pair correlation functions.

It remains to discuss how the corrected $g_{\alpha \beta}(r)$ are to be obtained. This is the content of the next section.

\section{CORRECTION FOR THE PAIR DISTRIBUTION FUNCTION}

Using Eq. (16), we first rewrite (36) in the form

$\Delta C_{\alpha \beta}(r) \approx-\beta \varphi_{\alpha \beta}^{(1)}+\Delta G_{\alpha \beta}(r)-\ln \left[g_{\alpha \beta}(r) / g_{\alpha \beta}^{(0)}(r)\right]$.

With this implementation of the HNC-type approximation, (37) defines the direct correlation function difference $\Delta C_{\alpha \beta}$ in terms of $\Delta G_{\alpha \beta}$ and known quantities. A second set of relations between the Fourier transform of these difference functions can be obtained from Eqs. (23) and their reference system counterparts. This is

$$
\begin{aligned}
\Delta \tilde{G}_{11}(k)= & W(k)^{-1}\left\{\rho_{1}^{-1}\left[1+\rho_{2} \tilde{G}_{22}^{(0)}(k)\right][1-W(k)]\right. \\
& \left.-D_{G}^{(0)}(k) \Delta \tilde{C}_{11}(k)\right\}, \\
\Delta \tilde{G}_{12}(k)= & W(k)^{-1}\left\{\tilde{G}_{12}^{(0)}(k)[1-W(k)]\right. \\
& \left.+D_{G}^{(0)}(k) \Delta \tilde{C}_{12}(k)\right\}, \\
\Delta \tilde{G}_{22}(k)= & W(k)^{-1}\left\{\rho_{2}^{-1}\left[1+\rho_{1} \tilde{G}_{11}^{(0)}(k)\right][1-W(k)]\right. \\
& \left.-D_{G}^{(0)}(k) \Delta \tilde{C}_{22}(k)\right\},
\end{aligned}
$$

where

$$
\begin{aligned}
W(k) \equiv & 1-\sum_{\alpha, \beta}\left[\delta_{\alpha \beta}+\rho_{\alpha} \tilde{G}_{\alpha \beta}^{(0)}(k)\right] \rho_{\beta} \Delta \tilde{C}_{\alpha \beta}(k) \\
& +\rho_{1} \rho_{2} D_{G}^{(0)}(k)\left[\Delta \tilde{C}_{11}(k) \Delta \bar{C}_{22}(k)-\Delta \bar{C}_{12}(k)^{2}\right],
\end{aligned}
$$


and $D_{G}^{(0)}(k)$ is defined as in (22). Equations (37) and (38) constitute a set of coupled nonlinear equations for the unknowns $\Delta G_{\alpha \beta}$. They are, in effect, a generalization of the coupled HNC integral equations for binary mixtures and can be solved in an analogous fashion, i.e., by iteration until selfconsistency is achieved. As in the one-component case, ${ }^{2}$ it will be more practical to solve these equations for the corrected $g_{\alpha \beta}(r)$ in the form

$$
H_{\alpha \beta}(r)=\ln \left[g_{\alpha \beta}(r) / g_{\alpha \beta}^{(0)}(r)\right]+\beta \varphi_{\alpha \beta}^{(1)}(r) .
$$

Equations (37) and (38) can be appropriately rewritten for this purpose. ${ }^{2}$ Though the equations are lengthy in appearance, they offer no unusual problems for numerical solution.

Even so, a simpler solution may be desirable, even if at the cost of an additional approximation. Evidently, the equations are solved if the $\Delta C_{\alpha \beta}$ are known. For these corrections, we may take the lowest-order contribution

$$
\Delta C_{\alpha \beta}(r) \approx-\beta \varphi_{\alpha \beta}^{(1)}(r),
$$

so that Eq. (37) immediately gives

$$
g_{\alpha \beta}(r) \approx g_{\alpha \beta}^{(0)}(r) \exp \left[\Delta G_{\alpha \beta}(r)\right],
$$

while $\Delta G_{\alpha \beta}$ in the exponent is determined from (38) using the approximation (41). This result, previously unpublished, ${ }^{14}$ is the generalization for mixtures of the perturbation correction proposed in Ref. 1.

\section{APPENDIX}

The variational properties of the last term of Eq. (28) are of particular interest, since the absence of this term from the approximation (30) leads to thermodynamic inconsistencies. Put

$$
\begin{aligned}
\beta \Delta A^{\prime} / N= & -\frac{1}{2} \rho \int_{0}^{1} d \xi \int d \mathbf{r} \sum_{\alpha, \beta} x_{\alpha} x_{\beta} \Delta B_{\alpha \beta} \\
& \times(r ; \xi) \partial \Delta G_{\alpha \beta}(r ; \xi) / \partial \xi=-\frac{1}{2} \rho \int_{0}^{1} d \xi \int d \mathbf{r} \\
& \times \sum_{\alpha, \beta} x_{\alpha} x_{\beta} B_{\alpha \beta}(r ; \xi) \partial g_{\alpha \beta}(r ; \xi) / \partial \xi \\
& +\frac{1}{2} \rho \int d \mathbf{r} \sum_{\alpha, \beta} x_{\alpha} x_{\beta} B_{\alpha \beta}^{(0)}(r) \Delta G_{\alpha \beta}(r) .
\end{aligned}
$$

We assume $B_{\alpha \beta}(r ; \xi)$ and $B_{\alpha \beta}^{(0)}(r)$ to be functionals of $g_{\alpha \beta}(r ; \xi)$ and $g_{\alpha \beta}^{(0)}(r)$, respectively, and allow the pair distribution functions to vary. The effect on $\Delta A^{\prime}$ is

$$
\begin{aligned}
\delta\left(\beta \Delta A^{\prime} / N\right)= & -\frac{1}{2} \rho \int_{0}^{1} d \xi \int d \mathbf{r} \sum_{\alpha, \beta} x_{\alpha} x_{\beta}\left[\delta B_{\alpha \beta}(r ; \xi) \partial g_{\alpha \beta}(r ; \xi) / \partial \xi\right. \\
& \left.+B_{\alpha \beta}(r ; \xi) \partial \delta g_{\alpha \beta}(r ; \xi) / \partial \xi\right]+\frac{1}{2} \rho \int d \mathbf{r} \sum_{\alpha, \beta} x_{\alpha} x_{\beta}\left[\delta B_{\alpha \beta}^{(0)}(r) \Delta G_{\alpha \beta}(r)+B_{\alpha \beta}^{(0)}(r) \delta g_{\alpha \beta}(r)-B_{\alpha \beta}^{(0)}(r) \delta g_{\alpha \beta}^{(0)}(r)\right] \\
= & -\frac{1}{2} \rho \int d \mathbf{r} \sum_{\alpha, \beta} x_{\alpha} x_{\beta}\left[\Delta B_{\alpha \beta}(r) \delta g_{\alpha \beta}(r)-\Delta G_{\alpha \beta}(r) \delta B_{\alpha \beta}^{(0)}(r)\right] \\
& -\frac{1}{2} \rho \int_{0}^{1} d \xi \int d \mathbf{r} \sum_{\alpha, \beta} x_{\alpha} x_{\beta}\left[\frac{\delta B_{\alpha \beta}(r ; \xi)}{\delta g_{\alpha \beta}(r ; \xi)} \frac{\partial g_{\alpha \beta}(r ; \xi)}{\partial \xi}-\frac{\partial B_{\alpha \beta}(r ; \xi)}{\partial \xi}\right] \delta g_{\alpha \beta}(r ; \xi)
\end{aligned}
$$

after an integration by parts. The integrand of the last term in (A2) vanishes, and we get

$$
\begin{aligned}
\delta\left(\beta \Delta A^{\prime} / N\right)= & -\frac{1}{2} \rho \int d \mathbf{r} \sum_{\alpha, \beta} x_{\alpha} x_{\beta}\left[\Delta B_{\alpha \beta}(r) \delta g_{\alpha \beta}(r)\right. \\
& \left.-\Delta G_{\alpha \beta}(r) \delta B_{\alpha \beta}^{(0)}(r)\right],
\end{aligned}
$$

from which it follows that

$$
\begin{aligned}
& \frac{\delta\left(\beta \Delta A^{\prime} / N\right)}{\delta g_{\alpha \beta}(r)}=-\frac{1}{2} \rho x_{\alpha} x_{\beta} \Delta B_{\alpha \beta}(r), \\
& \frac{\delta\left(\beta \Delta A^{\prime} / N\right)}{\delta g_{\alpha \beta}^{(0)}(r)}=\frac{1}{2} \rho x_{\alpha} x_{\beta} \Delta G_{\alpha \beta}(r) \frac{\delta B_{\alpha \beta}^{(0)}(r)}{\delta g_{\alpha \beta}^{(D)}(r)} .
\end{aligned}
$$

With this result, the contribution of $\Delta A^{\prime}$ to the derived thermodynamic quantities is easily determined. For the internal energy, for example, we get

$$
\frac{\partial\left(\beta \Delta A^{\prime} / N\right)}{\partial \beta}=\int d \mathbf{r} \sum_{\alpha, \beta} \frac{\delta\left(\beta \Delta A^{\prime} / N\right)}{\delta g_{\alpha \beta}(\boldsymbol{r})} \frac{\partial g_{\alpha \beta}(r)}{\partial \beta}
$$

$$
\begin{aligned}
& +\int d \mathbf{r} \sum_{\alpha, \beta} \frac{\delta\left(\beta \Delta A^{\prime} / N\right)}{\delta g_{\alpha \beta}^{(0)}(r)} \frac{\partial g_{\alpha \beta}^{(0)}(r)}{\partial \beta} \\
= & -\frac{1}{2} \rho \int d \mathbf{r} \sum_{\alpha, \beta} x_{\alpha} x_{\beta} \Delta B_{\alpha \beta}(r) \frac{\partial g_{\alpha \beta}(r)}{\partial \beta} \\
& +\frac{1}{2} \rho \int d \mathbf{r} \sum_{\alpha, \beta} x_{\alpha} x_{\beta} \Delta G_{\alpha \beta}(r) \frac{\partial B_{\alpha \beta}^{(0)}(r)}{\partial^{j} \beta},
\end{aligned}
$$

which was used in Eq. (33). It is to be noted that only one of the terms in (A6) contains $\Delta B_{\alpha \beta}$ explicitly. When $\Delta A^{\prime}$ is neglected, however, both terms of (A6) are dropped from Eq. (33). This results not only in the approximate $g_{\alpha \beta}(r)$ in (34), a consequence of the missing first term of (A6), but leads also to the appearance of an additional term in (33), the negative of the second (A6) term. That is to say, Eq. (34) with the approximate $g_{\alpha \beta}(r)$ is not the same as the $\beta$ derivative of Eq. (30). 
${ }^{1}$ F. Lado, Phys. Rev. 135, A1013 (1964).

${ }^{2}$ F. Lado, Phys. Rev. A (to be published).

${ }^{3}$ T. Morita and K. Hiroike, Prog. Theor. Phys. 23, 1003 (1960).

${ }^{4}$ M. S. Green, J. Chem. Phys. 33, 1403 (1960).

${ }^{5}$ J. M. J. van Leeuwen, J. Groeneveld, and J. DeBoer, Physica (Utr.) 25, 792 (1959); E. Meeron, J. Math. Phys. 1, 192 (1960); G. S. Rushbrooke, Physica (Utr.) 26, 259 (1960); L. Verlet, Nuovo Cimento 18, 77 (1960).

${ }^{6}$ P. J. Leonard, D. Henderson, and J. A. Barker, Trans. Faraday Soc. 66, 2439 (1970); G. A. Mansoori and T. W. Leland, Jr., J. Chem. Phys. 53, 1931 (1970).

${ }^{7}$ T. W. Leland, J. S. Rowlinson, G. A. Sather, Trans. Faraday Soc. 64, 1447 (1968); T. W. Leland, J. S. Rowlinson, G. A. Sather, and I. D. Watson, Trans. Faraday Soc. 65, 2034 (1969).
${ }^{8}$ J. S. Rowlinson, Liquids and Liquid Mixtures (Butterworths, London, 1969), 2nd ed., Chap. 9.

'J. K. Percus and G. J. Yevick, Phys. Rev. 110, 1 (1958).

${ }^{10}$ J. L. Lebowitz, Phys. Rev. 133, A895 (1964); G. J. Throop and R. J. Bearman, J. Chem. Phys. 42, 2838 (1965).

"A. A. Broyles, S. U. Chung, and H. L. Sahlin, J. Chem. Phys. 37, 2462 (1962); D. Levesque, Physica (Utr.) 32, 1985 (1965).

${ }^{12}$ D. D. Carley, Phys. Rev. 136, A127 (1964); J. F. Springer, M. A. Pokrant, and F. A. Stevens, Jr., J. Chem. Phys. 58, 4863 (1973).

${ }^{13}$ S. A. Rice and P. Gray, The Statistical Mechanics of Simple Liquids (Wiley, New York, 1965), Chap. 2.

${ }^{14}$ F. Lado, Ph.D. thesis, University of Florida, 1964 (unpublished). 